\title{
Traumatic brain injury patients: does frontal brain lesion influence basic emotion recognition?
}

\author{
Ana Teresa Martins ${ }^{1}$, Luís Faísca ${ }^{1}$, Francisco Esteves ${ }^{2}$, Angélica Muresan ${ }^{1}$, Mariline Gomes \\ Justo $^{2}$, Claudia Simão ${ }^{2}$, and Alexandra Reis ${ }^{1}$ \\ 1 - Universidade do Algarve, Faro, Algarve, Alg, Portugal \\ 2 - Instituto Universitário de Lisboa, Lisboa, Lx, Portugal
}

\begin{abstract}
Adequate emotion recognition is relevant to individuals' interpersonal communication. Patients with frontal traumatic brain injury (TBI) exhibit a lower response to facial emotional stimuli, influencing social interactions. In this sense, the main goal of the current study was to assess the ability of TBI patients in recognizing basic emotions. Photographs of facial expressions of five basic emotions (happiness, sadness, fear, anger, and surprise) were presented to 32 TBI patients and 41 healthy controls. Emotion recognition was measured by accuracy and reaction time. Overall performance of the TBI group was poorer than control group for emotion recognition, both in terms of accuracy and reaction time. It is suggested that TBI patients show impairment on emotion recognition, and this relation seems to be moderated by the lesion localization. Keywords: emotion recognition, basic emotions, TBI patients.
\end{abstract}

Received 5 September 2011; received in revised form 5 November 2011; accepted 29 November 2011. Available online 29 December 2011

\section{Introduction}

Changes in emotional and social behavior are easily associated with distinct neurological disturbances. Research focused on frontal traumatic brain injury (TBI), particularly in medial and orbitofrontal regions, has shown that one of the behavioral disturbances associated with these brain lesions is emotional deficits that influence social behavior (McDonald, 2005; Kendall \& Terry, 1996). Usually, these alterations are emotional lability, disinhibition, inappropriate behaviors, personality changes and irritability (Ardila, 2008). However, these emotional disabilities are not always associated with cognitive or executive disorders (Milders, Fuchs, \& Crawford, 2003) but appear to be related to the difficulty that TBI patients have in processing emotions as well as making appropriate social judgments (McDonald, 2005; Milders, Fuchs, \& Crawford, 2003). This impairment is particularly revealed in experimental paradigms that

Ana Teresa Martins, Luís Faisca, Angélica Muresan and Alexandra Reis, Departamento de Psicologia, Faculdade de Ciencias Humanas e Sociais da Universidade do Algarve (UALG). Francisco Esteves, Mariline Gomes Justo and Claudia Simão, Departamento de Psicologia Social e das Organizações (ISCTE), Instituto Universitário de Lisboa. Correspondence regarding this article should be directed to: Ana Teresa Martins, Departamento de Psicologia, FCHS, Universidade do Algarve, Campus de Gambelas - Faro Portugal. E-mail: atmartins@ualg.pt assess emotional processing through facial expressions, body posture and contextual parameters (Bornhofen \& McDonald, 2008).

Prigatano and Pribman (1982) used, for the first time, a visual facial recognition paradigm and reported an association between frontal TBI and impairments in emotion recognition. Their findings created new research interests, exploring deeper and with distinct paradigms the relation between frontal traumatic brain lesions and emotional states. Hence, it began to be noted that participants with TBI exhibited impairment for recognizing emotions but not for recognizing neutral facial expressions (Green, Turner, \& Thompson, 2004). Specifically, negative basic emotions like fear and anger are the most difficult for TBI patients to recognize (e.g., Spell \& Frank, 2000). Nevertheless, even with the finding of behavioral changes and impaired emotion processing in TBI patients, a great deal of divergence remains regarding not only which emotions are most impaired but also regarding the role played by each cerebral hemisphere and the cerebral localization of those brain injuries that impair adequate processing of each basic emotion.

Furthermore, it is commonly accepted in the current literature that there exists a functional hemispheric asymmetry regarding facial expression processing but a significant functional hemispheric asymmetry for processing of specific emotional states has not yet been determined. In this respect, two main theories have 
been suggested: (1) The Right Hemisphere Hypothesis, which states that the right hemisphere participates in processing of all emotions, both positive and negative (Morris \& Hopkins, 1993) and the (2) Valence Hypothesis, which states that the right hemisphere is relatively specialized to process negative emotions, whereas the left hemisphere is relatively specialized to process positive emotions (Borod et al. 1998; Canli et al., 1998; Lee et al., 2002). Tamietto et al. (2006) report that these two theories, which apply not only for TBI patients, differ only regarding the definition of "emotion" and that divergent conceptual perspectives regarding this matter led to distinct experimental approaches of emotion processing and thus to nonconcurring and scarcely comparable results.

Notwithstanding, there is also evidence from studies with bilateral brain-damaged patients pointing to concurring contribution of both theories in emotion processing from facial expressions (Adolphs et al., 2001, 2000, 1996; Davidson, 1992; Gainotti, 2001), thus suggesting cerebral interhemispheric cooperation. Adepts of this hybrid theory (e.g., Miller, 1986; Adolphs, 2002; Oatley \& Johnson-Laird, 1987; Plutchik, 1980; Braun et al., 2005; Kolb \& Taylor, 2000) defend that the emotional pathway circuits consist of distinct but interrelated neurocognitive components. Thus, different neural subtracts from both cerebral hemispheres are involved in expression and recognition of emotional states and their physiological correlates. In order to understand which specific neural subtracts are involved in emotion recognition from facial expressions, many studies of populations with neurological impairment used neuroimaging techniques and found a specific pathway circuit for emotion processing that engages frontotemporal regions including the amygdala (Bornhofen \& McDonald, 2008; Adolphs, 2002). Although these studies document that cerebral frontal cortex is a crucial neural subtract for emotion processing, it is also important to further understand whether this cerebral structure differentiates in distinct areas. For instance, there is evidence that patients with damage to the orbitofrontal cortex perform poorer on tasks of emotion recognition from facial and vocal expressions when compared to patients with lesions in other areas of the frontal cortex (Hornak, Rolls, \& Waidt, 1996). These authors also found that patients perform poorer when damages are to the right orbitofrontal cortex. Vuilleumier et al. (2001) also observed increased cerebral activation of the orbitofrontal cortex in patients performing emotion processing tasks.

As mentioned earlier, frontal and temporal brain areas seem to be more frequently damaged in traumatic brain lesions (Ietswaart et al., 2008) especially in those resulting from car accidents where frontal and temporal brain regions are most frequently injured. Due to the fact that emotional and behavioral disorders are a significant negative consequence of frontal and TBIs, this matter, broadly studied in different contexts and through various techniques, is far from being completely understood. Thus, our study intends to contribute to a better understanding of the implications of TBI in emotion processing. Furthermore, it is our goal to study the role played by each hemisphere and different areas of the frontal cortex in this process. Specifically, we intended to assess repercussions of frontal cortex lesions in recognition of five basic facial emotional expressions: happiness, sadness, fear, anger, and surprise. To accomplish this goal, we compared two distinct groups of participants: TBI and control group. We hypothesized that TBI patients should present more difficulty in recognition of basic emotions. In this way, it is expected that the control group will perform better than the TBI group in accuracy and speed of recognition of all five basic emotions.

Moreover, it was also our goal to analyze the localization of the brain lesion: hemispheric and frontal lobe lesion localization once it appears to define how emotion recognition is processed. To this end, we divided TBI participants into three different groups according to hemispheric lesion localization: left frontal injury, right frontal injury, and bilateral injury as well as in accordance with frontal lobe lesion localization: orbitofrontal, medial, and dorsolateral.

\section{Methods}

\section{Participants}

Before describing the participants of both groups, it is important to note that this study was conducted in accordance with the 1975 Declaration of Helsinki (as revised in Tokyo in 2004).

\section{TBI group}

Thirty-two TBI patients were assessed (20 males) with a mean age of 29.97 years $(S D=6.07)$ and mean number of years of education of $11.91(\mathrm{SD}=3.17)$. The posttraumatic mean number of years was 6.78 $(\mathrm{SD}=3.64)$. TBI patients' group was recruited from a database of a private neurosurgical clinical in Algarve $(n=28)$ and from the Neurosurgical Department of the Hospital of S. José, Lisbon. Inclusion criteria were (1) clinical diagnosis of frontal TBI, (2) within more than 12 months, and (3) posttraumatic amnesia $>24$ h. Exclusion criteria were as follows: (1) previous clinical history of psychiatric illness, (2) nonfrontal TBI, and (3) age $>40$ years old. From the 43 patients initially selected, 11 were excluded for two reasons: six abandoned the experiment arguing fatigue and physical indisposition and five were excluded for not showing images of significant frontal brain damage. In order to obtain a more conclusive characterization of their TBI, our sample of 32 TBI participants was submitted to a 
structural magnetic resonance imaging, all tested with a Philips 1.5 Intera Scanner and submitted to the same image protocol ${ }^{1}$.

Subsequently, two independent judges (a neurosurgeon and a neuroradiologist) rated neuroimaging data with the following grid criteria: frontal (anterior or posterior), frontal (orbitofrontal, medial, and dorsolateral), parietal (anterior or posterior), temporal (anterior or posterior), and occipital (anterior or posterior). Each of the lesions was characterized using the rules of abnormal focal signals (hypointense, hyperintense or mixed).

\section{Control group}

Forty-one healthy participants (25 males) along with an age- and education-matched TBI group were recruited to participate in this experiment. Exclusion criteria included the following: (1) neurological disease, (2) self-reported depression, and (3) past history of TBI. Mean age for the control subjects was 27.98 years (SD $=5.73$ ), and the mean number of years of education was $12.68(\mathrm{SD}=3.6)$

\section{Cognitive assessment}

Participants were submitted to cognitive assessment using several tests: (1) Raven's Progressive Matrices, (2) Wechsler Memory Scale, (3) Verbal Fluency Test, (4) Familiar Faces Recognition Test, and (5) Trail
Making Test-Part B. These tests were applied in the same order, allowing us to characterize participants' cognitive functions and also to be certain that variables other than those proposed by us could not explain the differences that we would eventually find between the two study groups (TBI/control).

Cognitive assessment was followed by the application of the Beck Depression Inventory (BDI) to check presence or absence of depressive symptoms both in control and TBI groups, because TBI patients are suggested to report high levels of depressive symptoms (van Zomeren \& van den Burg, 1995).

Table 2 reports means and standard deviation for both control and TBI groups for cognitive tests. This comparison allows us to confirm that groups did not differ for all cognitive variables, with the exception of the Familiar Faces Recognition Test where the control group scored higher. This difference was observed only when TBI participants had to identify the emotional state of the familiar face.

There was a statistically significant difference between groups for depressive symptoms. TBI group presented higher scores for BDI test, indicating low depressive symptoms compared to control group (see Table 2).

\section{Stimulus and apparatus}

We opted for the construction of the stimuli, not

Table 1. Distribution of TBI patients according to brain lesion localization

\begin{tabular}{lll}
\hline Brain lesion localization & $\boldsymbol{n}$ & \% \\
\hline Hemisphere & 8 & 25.00 \\
\hline Right anterior frontal (\%) & 10 & 31.25 \\
Left anterior frontal (\%) & 7 & 21.88 \\
Bilateral & & 34.38 \\
\hline Frontal & 11 & 9.38 \\
\hline Orbitofrontal & 3 & 56.25 \\
Medial & 18 & 6.25 \\
Dorsolateral & & 6.25 \\
\hline Others & 2 & 6.25 \\
\hline Left frontal/anterior temporal & 2 & 3.13 \\
Right frontal/anterior temporal & 2 & 1 \\
Frontal bilateral/anterior temporal & 25 \\
Right frontal/posterior occipital & & \\
\hline
\end{tabular}

TBI, traumatic brain injury.

${ }^{1}$ Sagittal Spin Echo T1 (22 cuts of $5 \mathrm{~mm}$ ), (2) Axial Turbo Spin Echo dual echo PD and T2 (22 cuts of 5 mm), (3) Axial Fluid Attenuated Inversion Recovery (Flair- 22 cuts of $5 \mathrm{~mm}$ ), (4) Axial Fast Field Echo (Gradient echo) T2 (22 cuts of $6 \mathrm{~mm}$ ), (5) Axial Diffusion Weight (Gradient) Echo Diffusion (20 cuts of $3 \mathrm{~mm}$ ), (6) Coronal Turbo Spin Echo T2 (25 cuts of $3 \mathrm{~mm})$, and (3) Axial 3D Fast Field Echo T1 (200 cuts of $0.6 \mathrm{~mm}$ ). 
Table 2. Sociodemographic, clinical, and cognitive characterization of TBI and controls groups (t-test)

\begin{tabular}{|c|c|c|c|c|c|}
\hline \multirow[b]{3}{*}{ Characterization } & \multicolumn{4}{|c|}{ Group } & \multirow[b]{3}{*}{$p$} \\
\hline & \multicolumn{2}{|c|}{ TBI } & \multicolumn{2}{|c|}{ Control } & \\
\hline & M & $\mathrm{SD}$ & M & $\mathrm{SD}$ & \\
\hline \multicolumn{6}{|l|}{ Sociodemographic } \\
\hline Age & 29.97 & 6.07 & 27.98 & 5.73 & .155 \\
\hline Years of education & 11.91 & 3.17 & 12.68 & 3.66 & .343 \\
\hline Years of TBI & 6.78 & 3.64 & N/A & $\mathrm{N} / \mathrm{A}$ & N/A \\
\hline \multicolumn{6}{|l|}{ Clinical } \\
\hline BDI & 2.13 & 0.75 & 1.17 & 0.38 & $<.001 * *$ \\
\hline \multicolumn{6}{|l|}{ Cognitive } \\
\hline RPM & 75.78 & 15.87 & 78.05 & 17.02 & .563 \\
\hline WMT & 87.09 & 12.06 & 91.29 & 8.79 & .090 \\
\hline TMT errors & 128.28 & 44.18 & 120.44 & 51.79 & .496 \\
\hline TMT time & 1.25 & 2.17 & 0.95 & 1.92 & .536 \\
\hline VFT & 22.39 & 5.58 & 24.20 & 5.39 & .062 \\
\hline Total FFRT & 7.13 & 2.28 & 9.39 & 1.07 & $.039 *$ \\
\hline Age perception & 3.03 & 1.12 & 3.36 & 1.23 & .534 \\
\hline Occupation & 2.01 & 0.89 & 2.65 & 2.30 & .563 \\
\hline Emotion & 2.09 & 0.27 & 3.38 & 1.02 & $.046 *$ \\
\hline
\end{tabular}

BDI, Beck Depression Inventory: (1) absence or minimal symptoms of depression, (2) mild depression, (3) moderate depression, and (4) severe depression; RPM, Raven Progressive Matrices: (I) 0-9: very low, (II-) 10-14: low, (II) 15-24: low, (III-) 25-49: medium, (III+) 50-74: normal, (IV) 75-84: good, (IV+) 85-89: good, (V) 90-94: very good, (V+) 95-100: very good; WM, Wechsler Memory Scale (minimum quotient range age from 20-44 years old: 40; maximum quotient age range from 20-44 years old: 136); TMT, Trail Making Test-Part B (performance mean time: $60 \mathrm{sec}$ ); VFT, Verbal Fluency Test: total number of words; FFRT, Familiar Faces Recognition Test (4 points for each subtest: age perception, occupation, and emotion. Total = 12 points); N/A, not applicable.

$* * p \leq .001, * p \leq .05$.

using the most widespread battery about emotion recognition of Ekman and Friesen (1975) because we wanted to design a battery not only to assess basic emotions but also social emotions. Because social emotions are not universal, we need to use Portuguese emotional expressions evaluated by Portuguese patients. In this line, we used the same actors for the paradigm of basic emotion recognition.

The task to measure participants' ability to decode specific emotional facial expressions was adapted from Kessler, Bayerl, Deighton, and Traue (2002). The basic emotion recognition task was designed to assess participants' ability to recognize five basic emotions as previously defined by Ekman (1992). Stimuli material used was previously validated (Martins \& Reis, 2007). Three actors were selected (one male and two females) who represented six different basic emotions: happiness, sadness, fear, anger, disgust and surprise, and one neutral facial expression per actor. Each of the three selected actors represented the five basic emotions under study (15 different black and white photos $(44.46 \mathrm{~cm} / 50 \mathrm{~cm})$ were taken). After a pre-test with 10 judges, we found a high pattern of concordance
(Krippendorff, 2004) in emotion processing for all three actors (alpha Krippendorff $\geq 70$ ) except in regard to the emotion "disgust" for one of them. Although high scores were generally obtained for this emotion, scores were not consistent for all three actors. Therefore, the basic emotion "disgust" was excluded from the final experiment. Each photo was presented six times to each participant, so each emotion was presented 18 times. In this way, each participant perceived 90 stimuli (3 actors x 5 emotions x 6 times) and the score of one was attributed to each right answer.

Presentation Software version 7.0 (http://nbs. neurobs.com/presentation) was installed on a computer (Samtron $73 \mathrm{v}$ ) and we also used a touch screen $(38 \mathrm{~cm})$ that permitted presentation of visual stimuli and labels and also registration of participants' time reactions. Participants' response accuracy was registered on a grid designed for this purpose.

The stimuli presentation began with a neutral photo (presented for $1500 \mathrm{~ms}$ ) to use the same actor in the neutral and in the emotional phase. This allowed us to control the physiognomic changes and allowed participants to interpret modifications in the actor's 
facial expression, followed by a photo of the same actor representing one of the five basic emotions (500 $\mathrm{ms}$ ). Each participant was instructed to categorize each presented emotion within a visual forced-choice answering format $(10,000 \mathrm{~ms})$. All five forcedchoice categories (happiness, sadness, fear, anger, and surprise) were presented in a randomized order. Participants chose the correct label by pressing the name with his/her indicator finger on the touch-screen. After participants made their choice or after the 10000 ms timed out, the next trial automatically began. In this force-choice experience the maximum time possible for each trial was $15,500 \mathrm{~ms}$.

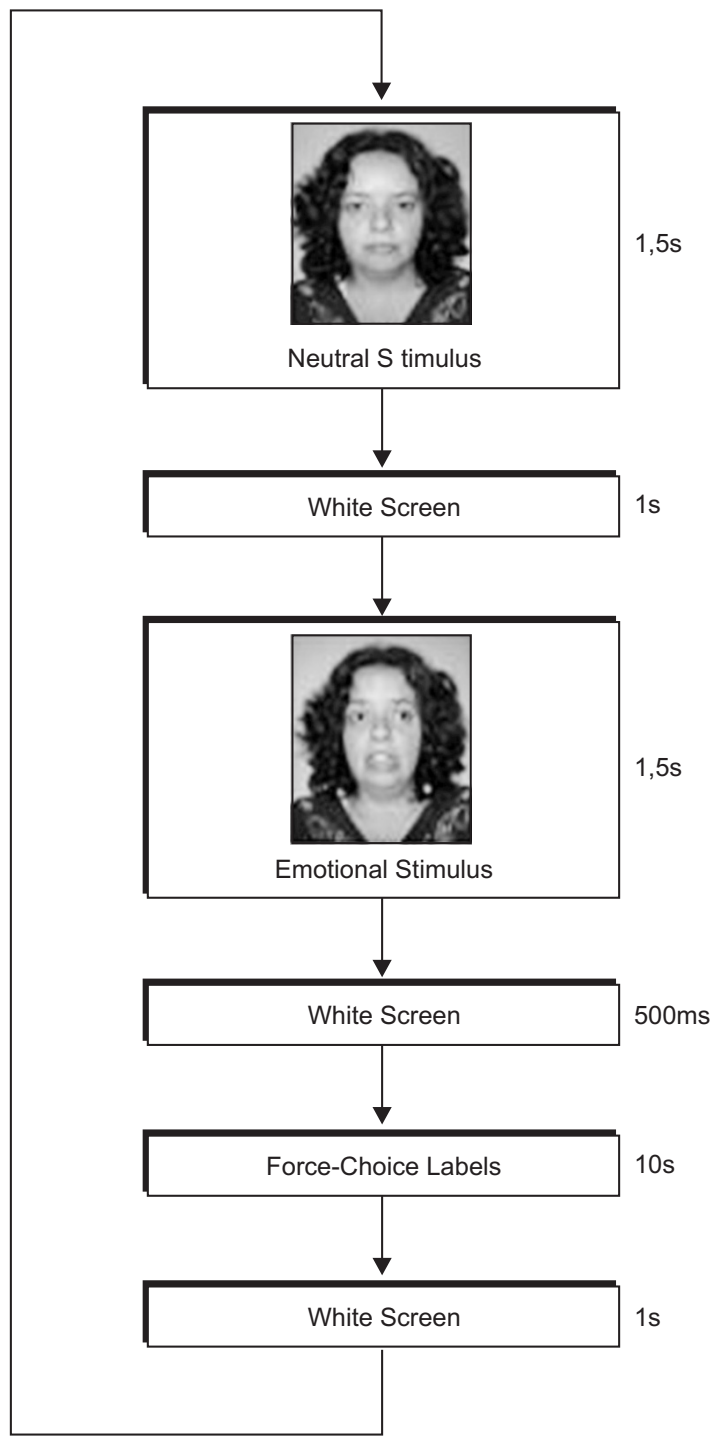

Figure 1. Scheme for basic emotional stimuli presentation.

\section{Procedure}

Both TBI and control groups were native Portuguese speakers and were individually assessed in a laboratory room in the Department of Psychology of the University of the Algarve.

Informed consent was obtained from all subjects after the nature of the study had been explained. In the beginning, participants completed a demographic questionnaire followed by application of the battery of cognitive tests. Afterwards, the basic emotion recognition task was applied. Participants were informed about the procedure as well as the meaning of each labeled emotion. A training session was performed by each participant before starting the emotion recognition task.

\section{Results}

\section{Analysis of the accuracy scores}

Accuracy was assessed by calculating the means of the correct answers for both groups (control and TBI). Next, the numbers of correct answers were also calculated using a d-prime $\left[d^{\prime}=\mathrm{Z}(\mathrm{H})-\mathrm{Z}(\mathrm{F})\right]$ performance discrimination model. This model allowed calculation and deletion of raw performance false alarms. The performance means were transformed into percentages. These percentages were analyzed using repeated-measures ANOVA. A factor group (TBI vs. control) and a factor emotion (emotion: happiness vs. sadness vs. fear vs. anger vs. surprise) were considered. Group was entered as a between-subjects factor and recognition of facial emotional expressions were entered as a within-subjects factor. The results reported a significant main effect for group $[F(1,71)=22.11, p<$ $\left..001, \eta_{p}{ }^{2}=.229\right]$ and for emotion $[F(4,292)=40.82, p<$ $\left..001, \eta_{p}{ }^{2}=.363\right]$. TBI patients presented lower accuracy scores when compared to control participants. No significant interaction effect between group and emotion was found $\left[F(4,292)=1.13, p=.341, \eta_{p}{ }^{2}=.016\right]$.

A post-hoc Tukey comparison analysis showed significant differences for the following emotions between groups: sadness $(p<.001)$, fear $(p<.001)$, anger $(p<.001)$, and surprise $(p<.01)$.

\section{Analysis of the reaction times}

Analysis of the reaction times (in $\mathrm{ms}$ ) of correct responses was analyzed to assess speed of response in recognizing emotions. A factor group (TBI vs. control) and a factor emotion (happiness vs. sadness vs. fear vs. anger vs. surprise) were considered in repeated-

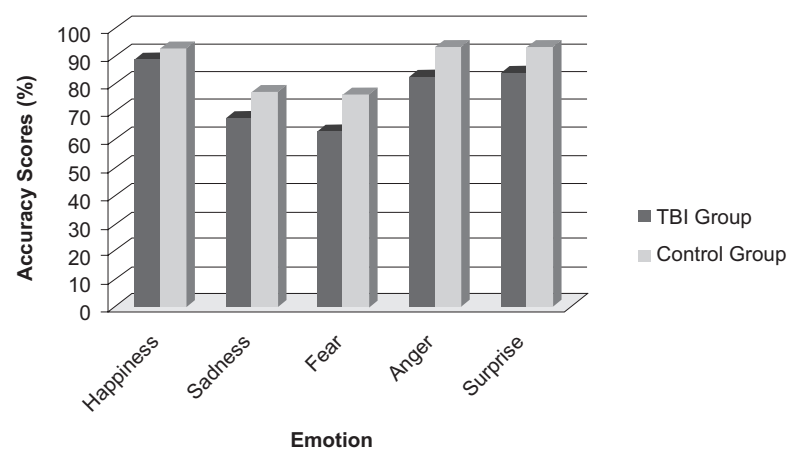

Figure 2. Accuracy Scores (\%) in each emotion for the two groups (control/TBI). 
measures ANOVA model. The group was entered as a between-subjects factor and recognition of facial emotional expressions was entered as a within-subjects factor. The results showed a significant main effect of group $\left[F(1,71)=9.36, p<.01, \eta_{p}{ }^{2}=.116\right]$ and of emotion recognition $\left[F(4,292)=13.56, p<.001, \eta_{p}{ }^{2}=\right.$ .160]. A significant interaction between group and speed of response was also found $[F(4,292)=3.22, p<.05$, $\left.\eta_{p}{ }^{2}=.043\right]$, which revealed that TBI patients compared to control group presented longer reaction times in their responses to sadness, fear, anger and surprise, particularly regarding these two last emotions.

A post-hoc Tukey comparison revealed significant differences between groups in the speed of emotion recognition: TBI patients presented longer reaction times in recognizing anger $(p<.01)$, surprise $(p<.001)$, and fear $(p<.05)$.

\section{Analysis of the TBI subgroups according to hemispheric lesion localization}

To analyze emotion recognition in TBI patients, a factor lesion localization (right vs. left vs. bilateral) and a factor emotion (happiness vs. sadness vs. fear vs. anger vs. surprise) were considered for an ANOVA model. A significant main effect for TBI group $[F(2,29)=3.33, p$ $\left.<.05, \eta_{p}^{2}=.209\right]$ and for emotion accuracy scores $[F(4$, $\left.116)=15.87, p<.001, \eta_{p}{ }^{2}=.340\right]$ was observed. TBI bilateral lesion participants presented the lowest scores for emotion recognition. No significant interaction effect was verified between TBI group and emotion recognition $\left[F(4,116)=0.53, p=.848, \eta_{p}^{2}=.034\right]$.

A post-hoc Tukey analysis revealed differences between TBI groups in emotion recognition scores: happiness (TBI left lesion group: $M=92.41, S D=$ 10.63, $S E M=1.06$; TBI bilateral lesion group: $M=$ 78.08, $S D=23.08, S E M=3.29 ; p<.05)$, anger $(\mathrm{TBI}$ left lesion group: $M=89.97, S D=13.15, S E M=1.31$; TBI bilateral lesion group: $M=74.55, S D=21.52, S E M$ $=3.07 ; p=.051$ ), and surprise (TBI left lesion group: $M=91.82, S D=15.54, S E M=1.56$; TBI bilateral lesion group: $M=73.14, S D=22.81, S E M=3.25$; $p$ $<.05)$. The longest reaction times were observed in the

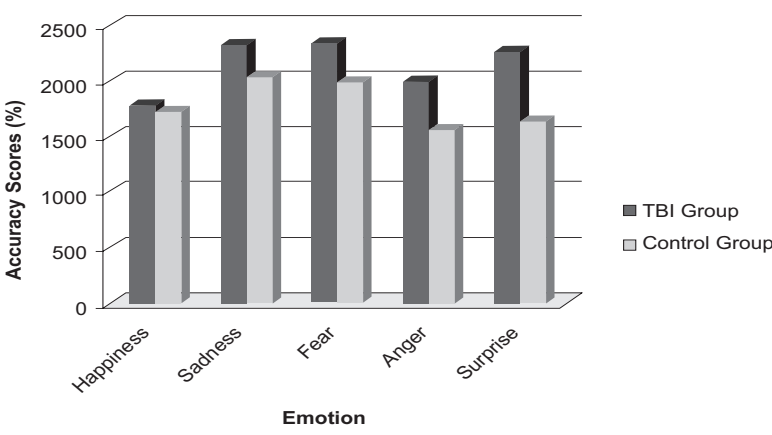

Figure 3. Reaction Times (ms) in each emotion for the two groups (control/TBI). bilateral frontal TBI group for all basic emotions in the study followed by the right frontal TBI group, although these differences were not significant.

\section{Analysis of the frontal lobe lesion localization}

In order to analyze differences in emotion recognition according to brain lesion localization, 32 TBI participants were distributed into three subgroups: orbitofrontal $(n=11)$, medial $(n=3)$, and dorsolateral ( $n=18)$ lesion. Statistically significant differences for accuracy were not observed. Mean differences in reaction time were compared according to KruskalWallis nonparametric statistics. A statistically significant difference was reported for the total mean reaction time in emotion recognition $(\mathrm{H}=7.232, p<.05)$. Longer reaction times were verified in orbitofrontal TBI subgroup followed by medial TBI subgroup and then by dorsolateral TBI group.

A potential association was also analyzed between the mild depressive symptoms revealed by the TBI group and their performance on basic emotion recognition task. Our results did not show any significant association in this respect $(r=0.122 ; p=$ 0.507 ), allowing us to infer that the mild depressive symptoms found in TBI patients did not influence, in any way, their performance on this task.

\section{Discussion}

It is broadly stated in the current literature that behavioral disorders found in TBI patients, particularly in those with damage to the frontal cortex, are associated with basic emotion recognition impairment (e.g., Kendall \& Terry, 1996; McDonald, 2005; Prigatano \& Pribram 1982; Green et al., 2004). Underlying this background, we asked a sample of frontal TBI patients to recognize five basic emotional states (happiness, sadness, anger, fear and surprise) from photos representing faces expressing certain emotional states.

Overall, our results suggest an impairment of TBI patients to recognize basic emotions except for happiness. In other words, the TBI group compared to controls presented lower performance in recognizing four basic emotions: sadness, fear, anger, and surprise. This difficulty in emotion recognition has already been documented by previous studies. Accuracy scores are lower and reaction times are higher in TBI participants, particularly for negative emotions (e.g., Croker \& McDonald, 2005; Green et al., 2004). Happiness recognition engages less sophisticated facial processing, first due to the fact that this emotion is transculturally known as represented by the stereotype of smile and second because, among all basic emotions, happiness is more innate and universal. In general, a lack of differences between clinical and nonclinical populations is documented in the literature in tasks that require processing of this specific basic emotion (Bornhofen \& McDonald, 2008). 
In contrast, accurate recognition of negative emotions requires a more sophisticated stimuli processing, thus engaging nonspecific frontal brain areas but is dependent on the interconnection of the frontal cortex, in its entirety, with multiple other brain areas among which are the temporal cortex and amygdala. In line with these findings, we consider that the difficulty exhibited by our TBI participants in recognizing negative emotions cannot be fully attributed to a specific frontal brain lesion but should be related to the whole frontal cortex and its interconnections. Functional neuroimaging studies (e.g., Phillips et al., 1998; Hennenlotter et al., 2004) have shown activation not only of the prefrontal cortex but also in amygdala in tasks involving fear emotion. Johnston et al. (2001) proposed a neural pathway circuit for emotion recognition and suggested that a lesion with a mere $10 \%$ extension of the frontal lobe is sufficient to compromise the pathway circuit, thus leading to a general impairment in recognition of negative emotions (anger, fear, disgust and sadness) when compared with recognition of positive emotions. In a specific analysis, we also observed that both TBI and control subjects obtained lower scores for fear recognition when compared to the other three negative emotions. According to Ekman and Friesen (1987), fear recognition is a more difficult process than recognition of any other basic emotion. Corden et al. (2006) showed that $8.8 \%$ from a sample of 341 healthy subjects had difficulties in fear emotion recognition from facial expressions. Furthermore, other authors (e.g., McDonald, 2005) emphasize that this percentage tends to increase in patients with damage to the frontal cortex and amygdala, which we also verify in our TBI sample.

Considering as well the discussion about whether emotion processing is lateralized and if that lateralization depends on the emotion content (Alves, Fukusima, \& Aznar-Casanova, 2008; Miller, 1986; Adolphs, 2002; Oatley \& Johnson-Laird, 1987; Plutchik, 1980), we divided our sample according to brain lesion localization and created TBI subgroups. The results suggested a poorer performance in emotion recognition for frontal bilateral TBI patients followed by right hemisphere lesion subgroup. Thus, these results seem to support the interhemispheric cooperation theory that postulates that both right and left hemispheres cooperate in basic emotion recognition.

Another important issue in the discussion about the relation between frontal cortex and emotion processing is that there will be a more specific region within the frontal lobe dedicated to this process. In this context, orbitofrontal lesions compared to other frontal brain lesions have been associated with poorer emotion recognition (Bornhofen \& McDonald, 2008; Hornak et al., 1996). In line with the evidence, our results showed a lower performance of the orbitofrontal TBI subgroup in emotion recognition: these participants presented longer reaction times when compared to other lesions of the TBI subgroups. It is well documented that the orbitofrontal cortex receives stimuli from all sensorial modalities (visual, auditory and somatosensory) and associates them with emotional states in order to give them emotional meaning. According to Hornak (2003), this is precisely what is required in emotion processing from facial expressions.

In summary, with this study we further corroborate that TBI patients present impairment in recognizing basic emotions. Furthermore, we also suggest that these differences in basic emotion recognition may not be directly associated with cognitive or executive disturbances. Most individuals with TBI are able to successfully plan and perform complex tasks of daily living. In our sample case, cognitive assessment revealed absence of executive disturbance. Regarding depressive symptoms, this is a common phenomenon in TBI patients. These depressive symptoms are primarily associated with family, work, and social reintegration (e.g., Eslinger, Parkinson, \& Shamay, 2002). We observed differences between TBI and control groups for depressive symptoms and this difference may be considered one limitation of the study; however, there is evidence that depressive symptoms do not directly influence facial emotion recognition. According to Kan et al. (2004), there is no correlation between depression and appropriate facial emotion recognition. In this regard, we sought to investigate if the mild depression of our TBI patients was correlated with their performance on basic emotion recognition task. Our results did not show any association between the variables. Thus, we infer that the depressive condition of TBI patients may not be related to their low performance on emotion recognition.

In summary and to conclude, we observed that TBI patients presented more difficulty in recognition of basic emotional states; however, we did not find differences between the TBI subgroups tested. Correct evaluation of the frontal lobe function can be a positive way to construct more efficient programs of cognitive rehabilitation. The main limitations of the study noted were the absence of more robust measures to assess the patient's injury (e.g., lesion volumetry) and more sophisticated evaluation (functional resonance) as well as behavioral methods.

\section{References}

Adolphs, R. (2001). The neurobiology of social cognition. Current Opinion in Neurobiology, 77, 231-239.

Adolphs, R. (2002). Neural systems for recognizing emotion. Current Opinion in Neurobiology, 15, 49-59.

Adolphs, R., Damasio H., Tranel D., Cooper G., \& Damasio A. R. (2000). A role for somatosensory cortices in the visual recognition of emotion as revealed by three-dimensional lesion mapping. Journal of Neuroscience, 20, 2683-2690. 
Adolphs, R., Damasio, H., Tranel, D., \& Damasio, A. (1996). Cortical systems for the recognition of emotion in facial expressions. The Journal of Neuroscience, 16, 7678-7687.

Alves, N. T., Fukusima, S. S., \& Aznar-Casanova, A. J. (2008). Models of brain asymmetry in emotional processing. Psychology \& Neuroscience, 1(1), 63-66.

Ardila, A. (2008). On the evolutionary origins of executive functions. Brain and Cognition, 68, 92-99.

Bornhofen, C., \& McDonald, S. (2008). Emotion perception deficits following traumatic brain injury: A review of the evidence and rationale for intervention. Journal of International Neuropsychological Society, 14, 511-525.

Borod, J., Obler, L., Erhan, H., Grunwald, I., Cicero, B., Welkowitz, J., ... Whalen, J. (1998). Right hemisphere emotional perception: Evidence across multiple channels. Neuropsychology, 12, 446-458.

Braun, M., Traue, H., Frisch, S., Deighton, R., \& Kessler, H. (2005). Emotion recognition in stroke patients with left and right hemispheric lesion: Results with a new instrument - the FEEL Test. Brain and Cognition, 58(2), 193-201.

Canli, T., Desmond, J., Zhao, Z., Glover, G., \& Gabrieli, J. (1998). Hemispheric asymmetry for emotional stimuli detected with fMRI Neuroreport, 9, 3233-3239.

Crocker, V., \& McDonald, S. (2005). Recognition of emotion from facial expression following traumatic brain injury. Brain Injury, 19, 787-799.

Davidson, R. (1992). Anterior cerebral assymmetry and the nature of emotion. Brain and Cognition, 6, 245-268.

Ekman, P. (1992). Are there basic emotions? Psychological Review, 99, 550-553.

Ekman, P., \& Friesen, W. (1987). Universal and cultural differences in the judgements of facial expressions of emotion. Journal of Personality and Social Psychology, 53, 712-717.

Ekman, P., \& Friesen, W. (1976). Pictures of facial affect. Palo Alto, CA: Consulting Psychologists Press.

Gainotti, G. (2001). Disorders of emotional behaviour. Journal of Neurology, 248, 743-749.

Green, R., Turner, G., \& Thompson, W. (2004). Deficits in facial emotion perception in adults with recent traumatic brain injury. Neuropsychologia, 42, 133-141.

Hennenlotter, A., Schroeder, U., Erhard, P., Haslinger, B., Stahl, R., \& Weindl, A. (2004). Neural correlates associated with impaired disgust processing in pre-symptomatic Huntington's disease. Brain, 127(6), 1446-1453.

Hornak, J., Bramham, J., Rolls, E. T., Morris, R. G., O’Doherty, J. P., Bullock, R., \& Polkey, C. E. (2003). Changes in emotion after circumscribed surgical lesions of the orbitofrontal and cingulate cortices. Brain, 126(7), 1691-1712.

Hornak, J., Rolls, E., \& Wade, D. (1996). Face and voice expression identification in patients with emotional and behavioural changes following ventral frontal lobe damage. Neuropsychologia, 34, 247-261.

Ietswaart, M., Milders, M., Crawford, J., Currie, D., \& Scott, C. L. (2008). Longitudinal aspects of emotion recognition in patients with traumatic brain injury. Neuropsychologia, 46, 148-159.

Kan, Y., Mimura, M., Kamijima, K., \& Kawamura, M. (2004). Recognition of emotion from moving facial and prosodic stimuli in depressed patients. Journal of Neurological Neurosurgery Psychiatry, 75, 1667-1671.

Kendall, E., \& Terry, D. (1996). Psychosocial adjustment following closed head injury: A model for understanding individual differences and predicting outcome. Neuropsychological Rehabilitation, 6, 101-132.
Kessler, H, Bayerl, P. Deighton, R. M \& Traue, H, C. (2002). Facially expressed emotion labeling (FEEL), PCgestützter test zur emotionserkennung. Verhaltenstherapie \& Verhaltensmedizin, 23, 297-306.

Kolb, B., \& Taylor L. (2000). Facial expression, emotion, and hemispheric organization. In R. D. Lane, \& L. Nadel (Eds.) Cognitive neuroscience of emotion (pp. 62-83), Oxford: Oxford University Press.

Krippendorff, K. (2004). Content analysis. An introduction to its methodology. London: Sage Publications.

Lee, T., Liu, H., Hoosain, R., Liao, W., Wu, C., Yuen, K., ... Gao, J. (2002). Gender differences in neural correlates of recognition of happy and sad faces in human assessed by functional magnetic resonance imaging. Neuroscience Letters, 333, 13-16.

Martins, A., \& Reis, A. (2007). Validação de estímulos para construção de paradigmas para o estudo do reconhecimento de emoções. Provas de Aptidão e Capacidade Científica apresentadas na Universidade do Algarve (texto policopiado).

McDonald, S. (2005). Are you crying or laughing? Emotion recognition deficits after severe traumatic brain injury. Brain Impairment, 6, 56-67.

Milders, M., Fuchs, S., \& Crawford, J. (2003). Neuropsychological impairments and changes in emotional and social behaviour following traumatic brain injury. Journal of Clinical and Experimental Neuropsychology, 25, 157-172.

Miller, W. (1986). The neuropsychology of head injuries. In D. Wedding, A. Horton, \& J. Webster (Eds). The neuropsychology handbook: Behavioural and clinical perspectives (pp. 347-375). New York: Springer

Morris, R., \& Hopkins, W. (1993). Perception of human chimeric faces by chimpanzees: Evidence for a right hemisphere advantage. Brain and Cognition, 21, 111-122.

Oatley K., \& Johnson-Laird, P.N. (1987). Towards a cognitive theory of emotions. Cognition and Emotion, 1, 29-50.

Phillips, R. G., \& Le Doux, J. E. (1992). Differential contribution of amygdala and hippocampus to cued and contextual fear conditioning. Behavior Neuroscience, 106, 274-285.

Plutchik, R. (1980). A general psychoevolutionary theory of emotion. In R. Plutchik \& H. Kellerman (Eds.), Emotion: theory, research, and experience: Vol. 1. Theories of emotion (pp. 3-33). New York: Academic Press.

Prigatano, G. P., \& Pribram, K. H. (1982). Perception and memory of facial affect following brain injury. Perceptual and Motor Skills, $54,859-869$

Spell, L.A., \& Frank, E. (2000). Recognition of nonverbal communication of affect following traumatic brain injury. Journal of Nonverbal Behavior, 24(4), 285-300.

Tamietto, M., Adenzato, M., Geminiani, G., \& Gelder, B. (2007). Fast recognition of social emotions takes the whole brain: interhemispheric cooperation in the absence of cerebral asymmetry. Neuropsychologia, 45, 836-843.

Van Strien, J., \& Morpurgo, M. (1992). Opposite hemispheric activations as a result of emotionally threatening and nonthreatening words. Neuropsychologia, 30, 845-848.

van Zomeren, A. H., \& van den Burg, W. (1985). Residual complaints of patients two years after severe head injury. Journal of Neurology Neurosurgery and Psychiatry, 48, 21-28.

Vuilleumier, P., Armony, J., Driver, J., \& Dolan, R. (2001). Effects of attention and emotion on face processing in the human brain: An event-related fMRI study. Neuron, 30, 829-841. 